\title{
INFORMATION AND COMMUNICATION TECHNOLOGY DEMANDS: OUTCOMES AND INTERVENTIONS
}

\author{
Jean-François Stich \\ Lancaster University Management School, Lancaster University, Lancaster, UK \\ Samuel Farley \\ Management School, The University of Sheffield, Sheffield, UK \\ Cary L. Cooper \\ Manchester Business School, University of Manchester, Manchester, UK \\ Monideepa Tarafdar \\ Lancaster University Management School, Lancaster University, Lancaster, UK
}

Purpose - The purpose of this paper is to review four demands employees face when communicating through information and communication technologies (ICTs). We review the outcomes associated with each demand and discuss relevant interventions to provide a set of evidence-based recommendations.

Design/methodology/approach - This paper reviews the following demands associated with ICTs: response expectations, constant availability, increased workload and poor communication. We draw upon empirical research to highlight outcomes and intervention strategies, before discussing implications for research and practice.

Findings - The findings suggest that there are diverse outcomes associated with each demand. The outcomes were not inherently negative as evidence suggests that positive performance outcomes can arise from response expectations and constant availability, although they may be allied by health and wellbeing costs.

Practical implications - A number of practical strategies are described to help organizations address computer-mediated communication demands, including tailored training, organizational policies and role modelling. The paper also outlines suggestions for future research on the dark side of IT use.

Originality/value - This paper integrates four interrelated demands that employees can face when communicating through technology. We extend knowledge by analyzing interventions which enables a synthesis of implications for practice. 


\section{INTRODUCTION}

Many employees are reliant on information and communication technologies (ICTs) to communicate with colleagues, clients and partners. Actual data concerning the spread of ICTs across different industries is difficult to capture due to the evolving nature of work, but it is clear that communication technologies are highly prevalent. In 2009, 90 percent of U.K businesses had access to the internet (Office for National Statistics, 2009), whilst in the USA 62 percent of employees could be considered networked workers, a term coined to describe individuals who use the internet or email in their workplace (Madden \& Jones, 2008).

In this paper, we examine evidence relating to ICT working practices from an organizational effectiveness perspective. At present, the productivity gains that arise from ICTs (including efficient file sharing and travel cost savings) are well known (Palvalin, Lönnqvist and Vuolle, 2013), however research in this area has often focused on the benefits without signaling the risks. We argue that the effectiveness of utilizing ICTs for computer-mediated communication is debatable. This debate concerns "whether the tools used are risk-free, or at least do not produce undesirable 'sideeffects"' (Mano and Mesch, 2010, p. 67). In this respect, it has been argued that ICTs can be viewed as both a support and a demand for employees (Day, Scott and Kelloway, 2010). Day et al. (2012) empirically identified eight demands associated with ICTs, including ICT hassles, response expectations and constant availability. ICT demands can negatively impact on organizational effectiveness by directly affecting how employees function. For example, ICT hassles (e.g. computer crashes, viruses, slow internet speed) could inhibit task completion, while poor communication could promote misunderstanding and ineffective decision making.

This paper will review how four of the demands identified by Day et al (2012) impact on organizational effectiveness. The research builds on Day et al's (2012) empirical study by updating the evidence on ICT demand outcomes, but perhaps more centrally by reviewing interventions which have been developed to address each ICT demand. In conducting this review, we aim to provide a more transparent picture of how ICTs contribute to organizational effectiveness, while also presenting evidence that can inform the design of more effectual organizational interventions. The paper concludes with implications for research and practice.

\section{LITERATURE REVIEW}

Four of the demands identified by Day et al (2012) specifically link to the way ICTs are used for employee communication, these are: (1) the extent that employees are expected to respond quickly to computer-mediated communication; (2) the extent that ICTs enable constantly availability; (3) the use of ICTs to increase workload; and (4) poor communication. We examine each factor in turn to identify how these demands relate to organizational outcomes. For each factor we first analyze the organizational outcomes and then provide suggestions for interventions that can address them. After reviewing each communication demand we elaborate on interventions used to mitigate their impact in order to provide suggestions for managers and organizations. 


\subsection{Response Expectations}

\section{Organizational Outcomes}

Having to respond quickly to requests is not a demand specific to ICT or technology, indeed employees are often interrupted more in person (Van Solingen et al., 1998). ICT has however provided new opportunities for constant interruptions that ask for immediate responses. The phone has been interrupting employees for long enough (1998), but recent literature is now focusing on emails (e.g., Barber and Santuzzi, 2015) and instant messaging (e.g., Gupta et al., 2013) due to their increasing prevalence within organizations. Unlike phone calls, emails are supposed to be asynchronous and as such could be replied to whenever convenient. However, a study that investigated the responding habits of employees within a large UK company found that most emails were dealt with within six seconds of reception, with an average of 1min44s (Jackson et al., 2001). The fast pace at which incoming emails are checked (along with arrival notifications) can thus make them as disturbing as synchronous media (e.g. phone calls, instant messaging) (2001). Regarding the impact of such interruptions on performance, Mano and Mesch (2010) found that email interruptions led to enhanced work performance, perhaps because they "increase the acquisition of work-related information critical for getting the job done" (2010, p. 68). Yet this explanation only covers emails which contain critical information and typologies suggest that other interruptions, such as those transmitting systems information, might actually have the opposite effect (Addas and Pinsonneault, 2015).

The impact may also depend on the type of task at hand, as well as how performance is evaluated. An experimental study found that simple tasks were accomplished quicker and with greater accuracy than more complex tasks because of interruptions, as interruptions focused participants' attention on important cues (Speier et al., 2003). Therefore performance on simple or short tasks might be less negatively impacted by interruptions than performance on more complex or longer projects (Addas and Pinsonneault, 2015). Similarly, interruptions asking for an immediate response unrelated to the task at hand fragment attention and reduce time dedicated to primary task activities. This can potentially result in lowered performance, efficiency and work quality (2015). These negative effects can be further nuanced by contextual elements. An experimental study found that instant messages became more disruptive as the hierarchical level of the sender increased (Gupta et al., 2013). Individual variables might also moderate response expectations, as individuals who enjoy multitasking might be less negatively affected by instant message interruptions (Li et al., 2011). Furthermore, selfdisciplined individuals might be more capable of resisting interruptions, which enables them to maintain their productivity (Al-Dabbagh et al., 2014).

Interruptions not only fragment attention, they also make it harder for an individual to return to their primary task. Employees within a large UK organization took an average of 64 seconds to recover from an email interruption to reengage in their primary task (Jackson et al., 2001). Other studies have referenced recovery times as long as 15 minutes (Van Solingen et al., 1998). Given the time taken away from the primary task to deal with response 
expectations, it has been estimated that knowledge workers lose $4-5 \%$ or 28 minutes of their workday to interruptions (Gupta and Sharda, 2008), whilst more than ten interruptions per day can severely hinder worker productivity (Van Solingen et al., 1998).

Although interruptions that transmit information necessary for task completion might actually increase performance (Mano and Mesch, 2010) (as opposed to other interruptions) (Addas and Pinsonneault, 2015; Speier et al., 2003), these productivity gains may be counterbalanced by detrimental effects on mental and physical health (Barber and Santuzzi, 2015). Indeed, the 'workplace telepressure' created by response expectations was associated with higher physical and cognitive burnout, health-related absenteeism and poorer sleep quality (2015).

\section{Possible interventions}

In order to tackle interruptions and response expectations, researchers have made several suggestions. A frequent suggestion has been to increase the interval at which incoming messages are checked (Addas and Pinsonneault, 2015; Gupta et al., 2011; Jackson et al., 2001, 2006), although this does not apply to phone calls. It has been estimated that increasing the email checking frequency from 5 minutes to 45 minutes could save employees 13.16 minutes per day, which would have accounted for a $£ 10,000$ per day saving in the organization surveyed for the study (Jackson et al., 2006). Other researchers went as far as to suggest a checking frequency of between two and four times per day (Gupta et al., 2011; Kushlev and Dunn, 2015). Increasing the email checking frequency has been found to increase task coordination, perhaps because it increases certainty among recipients that their messages will be dealt with at a specific time (Dabbish and Kraut, 2006). Though increasing the email checking frequency can improve productivity and task coordination, it can also increase email overload, as checking emails whenever they arrive as opposed to in batch prevents the inbox piling up (2006).

As an additional strategy, employees could work on their email or instant message checking self-discipline (Addas and Pinsonneault, 2015; Al-Dabbagh et al., 2014). Although recipients are not the only ones to blame, as senders should also try to limit the number of interrupting messages they dispatch (Addas and Pinsonneault, 2015). This can be done by only contacting the relevant individual and by avoiding 'reply-to-all' messages (Jackson et al., 2003). If senders do not expect immediate responses, they could avoid synchronous messages such as instant messages or phone calls as they create stronger response expectations (Gupta et al., 2013). Managers need to be particularly aware that sending messages which are unrelated to their subordinates primary task can divert attention and reduce its final quality, especially if the task is more complex (Addas and Pinsonneault, 2015). Managers might also consider their subordinate's orientation towards multitasking and avoid interrupting employees who prefer to focus on a single task (Li et al., 2011).

On an organizational level, a "companywide awareness of interruptions' impact on productivity" (Van Solingen et al., 1998, p. 101) needs to be promoted by assessing the impact within a company (Al-Dabbagh et al., 2014) and by collectively discussing the results in order to create a more empathetic organizational culture (Barber and 
Santuzzi, 2015). Response expectations can also occur outside of working hours, thus blurring work-home boundaries and creating pressure for $24 / 7$ availability (Barber and Santuzzi, 2015). We now discuss the implications of constant availability.

\subsection{Constant Availability}

\section{Organizational Outcomes}

This widely researched and reported aspect of the work-related ICT culture concerns how ICTs make it possible for work to cross into the non-work domain (Currie \& Eveline, 2011; Diaz et al., 2012) and increase productivity expectations (Ayyagari et al., 2011; Tarafdar et al., 2007). A survey of Australian workers found that $38 \%$ of Australians check their emails during non-work hours and kept their mobile phones switched on (Weekend Australian, 2007). Moreover, in the UK it was recently reported that managers work an extra day each week in unpaid overtime, a finding that was attributed to the smartphone culture and 'tech pressure' (BBC News, 2014).

This constant connectivity materializes in corporate smartphones, such as BlackBerrys or remote access to work communication media (e.g. email). Although these devices and media have sometimes been described as an "electronic leash" tying employees to their workplaces (Boswell and OlsonBuchanan, 2007), studies have found that employees rarely resist the introduction of such technologies and even embrace it (Cavazotte et al., 2014; Mazmanian, 2013), feeling more satisfied with their jobs as a result (Diaz et al., 2012). Most are even eager to claim that they accepted the introduction of these technologies of their own free will and with no organizational pressure (Mazmanian, 2013), while some think it is an inherent necessity of their jobs (Waller and Ragsdell, 2012). These desires might actually emerge less from organizational demands and more from friends, family, industry or society in general which perpetuate or glorify this "always-on" culture (Matusik and Mickel, 2011). Indeed, employees often consider these devices and media as "empowering", and feel a greater sense of professionalism, productivity, autonomy and control when using them (Cavazotte et al., 2014). They also feel more productive and more able to multi-task due to having these technologies (Matusik and Mickel, 2011). Similarly, teleworkers - who mostly work outside the office - feel a greater sense of productivity (Bailey and Kurland, 2002) which is sometimes reflected by higher supervisor ratings and job performance (Gajendran and Harrison, 2007). Accordingly, availability was the only dimension of ICT demands that was positively associated with professional efficacy in Day and colleagues' framework (Day et al., 2012).

These positive organizational outcomes and employee perceptions however can come at a personal cost, making constant availability a "double-edged sword" (DénNagy, 2014). "Advantages that appear in the domain of work can require sacrifices in private life" (2014, p. 208) and studies have linked constant availability to increased work-life conflict and work-life imbalance (Derks et al., 2015; Diaz et al., 2012; Matusik and Mickel, 2011; Wright et al., 2014), outcomes which have been associated with job burnout (Wright et al., 2014). Although ICT facilitates these outcomes, individuals also share some of the responsibility (Mazmanian, 2013). Employees and especially managers who send messages outside working hours implicitly encourage constant availability by creating response expectations (Derks 
et al., 2015). Nevertheless, individuals who have a more positive view of this connectivity do not experience as much work-life conflict as those who view it negatively (Wright et al., 2014). Similarly, employees who are highly engaged experience less work-home interference (Derks et al., 2015), while employees who prefer to segment their home and work roles report greater psychological detachment (Park, Fritz \& Jex, 2011).

Qualitative studies have shown that employees are generally aware of these trade-offs when they choose to become available outside working hours (Cavazotte et al., 2014; Matusik and Mickel, 2011). Participants in Waller and Ragsdell's study (2012) and especially "middle and senior managers who appear to be the victims (and culprits) of a 24/7 culture of accessibility" (2012, p. 170) were clearly aware that these technologies had a negative impact on their lives outside work; yet they continued to use them. Paradoxically, employees often claim these technologies give them greater autonomy, while they remain aware that technologies also allow for increased organizational control and surveillance (Cavazotte et al., 2014) and even decrease autonomy in practice by reducing the ability to disengage from work (Mazmanian et al., 2013). As employees are often aware of these trade-offs, they cope with the demands created by $24 / 7$ availability by taking them less seriously or finding justifications for the choices they make (Cavazotte et al., 2014).

\section{Possible interventions}

Given that employees often choose to remain constantly available and embrace technologies that facilitate constant connectivity (Cavazotte et al., 2014; Mazmanian, 2013), they may feel empowered by these technologies and be more productive as a result (Matusik and Mickel, 2011). Therefore organizations might have a hard time protecting their workers from the resulting "self-inflicted work-life conflict" (Wright et al., 2014, p. 524). The ability to detach from work is however important for employee wellbeing as empirical evidence demonstrates that a lack of recovery from work is associated with negative health outcomes, including burnout and psychosomatic health complaints (Eden, 2001; Lundberg \& Lindfors, 2002).

In terms of organizational policies and culture, a main element to consider is whether to promote or react against norms of constant availability (Matusik and Mickel, 2011). If these norms were to be promoted, organizations should try to increase awareness of associated worklife issues (Diaz et al., 2012). For example, collective discussions could be held about the topic (Barber and Santuzzi, 2015) and support groups could be implemented where employees could share suggestions on best practice (Leonardi et al., 2010). Training interventions could even be designed to better prepare employees for the work-life conflicts that they might face once they adopt these technologies (Leonardi et al., 2010). Managers should also give special consideration to employees who are more vulnerable to the work-life conflict, such as older, less engaged, less tech-savy workers or those who view these norms negatively (Waller and Ragsdell, 2012; Wright et al., 2014).

If constant availability were to be fought, organizational policies that limit access to ICT at night and during weekends could be enforced (Barber and Santuzzi, 2015; Wright et al., 2014). More informally, organizations could circulate guidelines that explicitly discourage work communication beyond working hours 
(Wright et al., 2014). Managers should also make clear their expectations on constant availability (Derks et al., 2015). If managers want to limit interactions beyond working hours, they should act as role models by refraining from sending any such messages themselves. Otherwise they are likely to create response expectations and encourage the same "always-on" culture they wish to prevent (2015). Finally, a more subtle approach identified by Matusik and Mickel (2011) is to limit the use of corporate smartphones or similar devices to organizational-specific interactions. The authors state that when employees are encouraged to use devices for organizational and personal interactions, the organization is "encouraging the highest degree of responsiveness and accessibility" (Matusik and Mickel, 2011, p. 1023).

As response expectations expand beyond working hours creating pressures for constant availability, employees might experience increased workloads and longer work days. This changing nature of workload is now discussed.

\subsection{Workload}

\section{Organizational Outcomes}

When examining the impact of ICT on workload and its associated organizational outcomes, several questions arise. What is the contribution of ICT to the overall workload of employees? Has workload increased or decreased with the introduction of ICT? If so, under what circumstances? Regarding the initial question, one study found that interruptions from instant messaging systems led to a higher perceived workload (Gupta et al., 2013). Similarly, it has been found that receiving more emails increases the length of the workday and produces greater feelings of overload (Barley et al., 2011). In addition, studies have examined the volume of interactions by calculating the average time spent reading or sending emails in organizations. Jackson and colleagues (2006) found that employees spent 29 minutes reading emails each day, which cost their organization $£ 40,848$ per day or $£ 9.8$ million per year, on the basis of the hourly cost of work. These figures do not however take into account how reading these emails might have allowed the organization to save time and money elsewhere. For instance, it has been found that the more work-related emails received, the higher the work effectiveness (Mano and Mesch, 2010). Nevertheless, stress and distress were also higher because of more numerous work-related emails, leading the authors to state that "technology has provided excellent means for achieving competition driven goals. But insofar as it affects employees, it may have given rise to unexpected side-effects" (Mano and Mesch, 2010, p. 68).

Stress also arises from ICT communications in form of information overload. In particular, email overload which has been defined by Dabbish and Kraut (2006) as "users' perceptions that their own email use has gotten out of control because they receive and send more email than they can handle, find or process effectively" (2006, p. 431). Their study found that email overload led to reduced task coordination. This was the result not of the sheer volume of emails, but of email overload as subjectively perceived by individuals. On a more transverse level, perceived communication overload has been found to lead to perceived losses in productivity for individuals who depend highly on communication technology in their jobs (Karr-Wisniewski and Lu, 2010). Other 
workers might also be more at risk of email overload. Jackson et al. (2006) found that employees in geographically dispersed teams frequently returned to their inbox to find that emails had piled up whilst they were on the move or in meetings, thus leading to increased overload.

\section{Possible interventions}

Most studies have focused on interventions targeted at reducing email overload. Email overload is based not only on email volumes but also on users' ability to handle their emails (Dabbish and Kraut, 2006). Reducing feelings of email overload could be done by either "reducing the amount of incoming information" or "enhancing recipients' information processing capabilities" (Soucek and Moser, 2010, p. 1459). Regarding the former, studies have suggested that organizations should design and circulate email guidelines to encourage users to limit the amount of emails they send (Soucek and Moser, 2010). As informational emails sent by the organization contribute to email overload, these types of emails could instead be sent via alternative channels, such as an intranet or an RSS news feed "rather than sending email "blasts" that necessitate action by every employee" (Mark et al., 2012, p. 10).

In order to enhance users' abilities to deal with email overload, training interventions could be designed and implemented (Burgess et al., 2005; Jackson and Lichtenstein, 2011; Soucek and Moser, 2010). A comprehensive training intervention designed by Soucek and Moser (2010) consisted of improving people's ability to use email features such as filters, improving personal workflow and enhancing the quality of written emails. Trainers first demonstrated techniques such as email filtering or archiving and discussed effective email communication. Participants then took part in role-playing exercises in which they had to filter, categorize and respond to fictional emails designed to reflect common problems such as newsletters, spams or poorly written emails. After each exercise, participants received feedback from the trainer and discussed their actions with peers. In the final part, the trainer displayed examples of poorly written emails and debated recommendations for best practices. As a take-away activity, participants had to draft an email policy that they could circulate among their colleagues or team members. Following the training, participants had greater knowledge of email functions and applied them more in their jobs, which reduced strain and improved productivity. Although the volume of emails did not change posttraining, participants were able to cope with this volume because of enhanced information processing abilities.

Finally, organizations could try to "promote a culture with a high perception of email as a business critical tool" (Sumecki et al., 2011, p. 413), as more positive views towards email can also mitigate feelings of email overload (2011). Organizations could also assess information overload in order to help individuals who are most at risk of becoming overloaded (Karr-Wisniewski and Lu, 2010). Furthermore, technical solutions could be implemented by adding task management features in email applications (Bellotti et al., 2005), setting email applications to check incoming messages less frequently (Dabbish and Kraut, 2006) or even sharing the email volume with a personal assistant, a crowdsourced assistant or an algorithm (Kokkalis et al., 2013). 
As overload can also be the result of poorly formulated emails or misunderstandings (Jackson et al., 2006), we now discuss the organizational outcomes and interventions associated with poor communication.

\subsection{Poor Communication}

\section{Organizational Outcomes}

The literature suggests that there are two main ways in which employee and organizational functioning can be interrupted by poor communication. Firstly, individuals may misunderstand the messages they receive through ICTs which can produce frustration, stress (Day, Scott and Kelloway, 2010) and poor decisions (Jackson and Van den Hooff, 2012). Secondly when employees are subjected to online communications perceived as uncivil or aggressive it can produce detrimental individual-level and organizational-level outcomes, including reduced wellbeing and turnover intentions (Baruch, 2005; Giumetti, Hatfield, Scisco, Schroeder, Muth and Kowalski, 2013).

A significant body of literature has focused on how ICT produces understanding costs for employees (Daft and Lengel, 1986; Kock, 2004; Sproull and Kiesler, 1986). Byron (2008) suggests that the primary outcome of miscommunication via email is a reduction in the quality of interpersonal relationships, which are integral to organizational functioning. Although, Byron (2008) also speculated that miscommunication may inhibit task performance when positive feedback is interpreted as emotionally neutral by a subordinate. In similar vein, Friedman and Currall (2003) developed a framework that outlines how structural elements of email communication (including diminished feedback and excess attention) are particularly amenable to misunderstanding and conflict.

Although it is generally accepted that miscommunication impairs individual and team effectiveness, limited empirical study has focused on outcomes of computer-mediated misunderstandings. However, research has shown that when virtual teams are distributed, a degree of situation invisibility exists as communication partners are less aware of contextual factors in their partner's environment (Cramton, Orvis and Wilson, 2007). This has implications for virtual team outcomes as teams have reported lower social cohesion and satisfaction when situation invisibility exists (Cramton et al., 2007). When employees are less aware of their communication partner's environment, they are more likely to make dispositional attributions about negative partner behavior than situational attributions (Walther, Boos and Jonas, 2002). Dispositional attributions occur when behavior is attributed to an individual's personality, whereas situational attributions are made when behavior is believed to stem from a person's environment. Walther et al. (2002) found that unwarranted dispositional attributions were linked to lower task performance and lower relational attraction. These unwanted byproducts of distributed communication may be less problematic when communication is fairly infrequent and facilitates the completion of simple tasks. However, it has been suggested that even synchronous media, such as video conferencing should not be used for discussing controversial topics or creative ideas (Panteli and Dawson, 2001), as it encourages more formal communication whereby it is difficult to 
build chemistry and rapport.

Poor communication also encompasses negative interpersonal communication, including cyber incivility (Lim and Teo, 2009), cyberaggression (Weatherbee and Kelloway, 2006) and cyberbullying (Privitera and Campbell, 2009). Cyber incivility has been defined as "communicative behavior exhibited in computer-mediated interactions that violate workplace norms of mutual respect" (Lim and Teo, 2009, p.419). Several studies have demonstrated how cyber incivility can reduce organizational effectiveness by depleting employee resources. Giumetti et al. (2013) conducted a within subjects experiment whereby participants completed math tasks whilst interacting electronically with a supportive and an unsupportive supervisor. The results showed that individuals reported higher negative affect, lower energy and lower task performance in the uncivil condition. A field study identified similar findings, as experiencing day-specific cyber incivility was associated with greater distress on that work day which persisted until the following work day (Park, Fritz \& Jex, 2015).

Workplace cyberbullying has also been linked to detrimental outcomes. Cyberbullying differs from cyber incivility as it involves repeatedly experiencing negative behaviors from a more powerful perpetrator (Heatherington and Coyne, 2014). Cyberbullying has been associated with intention to quit (Baruch, 2005), frustration, anxiety and vengefulness (Hong, Chien-Hou, Hwang, $\mathrm{Hu}$ and Chen, 2014) and lower job satisfaction (Farley, Coyne, Sprigg, Axtell and Subramanian, 2015; Snyman and Loh, 2015).

A study by Porath and Pearson (2010) outlined the costs of offline incivility, which included decreased work effort, decreased attendance, reduced work quality, reduced organizational commitment, reduced time spent working due to worrying and increased turnover intentions. Yet online harassment may more readily undermine effectiveness because online acts are more visible which may affect an organizations reputation. For instance, in April 2014 it was reported that one in five British workers had criticized their boss on social networking sites (The Metro, 2014). Furthermore, cyber harassment can be anonymous and it can affect employees outside working hours, which can heighten fear of future harassment as experiencing unwanted messages from an unknown perpetrator produces greater ambiguity (Ford, 2013).

\section{Possible interventions}

Several interventions geared towards improving communication have involved employee training. Jackson, Burgess and Edwards (2006) administered a questionnaire to $\mathrm{Plc}$ employees to identify difficulties associated with sending and receiving emails. Sender training was then administered to employee groups within the organization which involved targeting emails, getting the message across and using an effective subject line. The results showed that there was a significant improvement in the overall quality of messages received by recipients as a result of the training. Most notably, the training has a significant impact on email clarity as recipients reported that emails were better written, easier to read and more to the point after training. Jackson and colleagues (2006) estimated that the training could save the company $£ 3,071$ per day or $£ 737,000$ per year thanks to the reduced time spent reading more 
efficient emails. Organizations that rely heavily on email communication should therefore consider implementing email training as these results suggest it may produce significant financial savings.

Organizations should also consider whether employees would benefit from video conferencing training. Panteli and Dawson (2001) reviewed video conferencing training in a multinational oil company to find that $90 \%$ of employees who received the training felt competent using video technology to communicate post-intervention.

When virtual teams are highly prevalent within organizations, managers should also consider implementing training for virtual team members. Rosen et al. (2006) surveyed 440 organizations to identify best practice in virtual team training, although more than $60 \%$ of the 440 organizations surveyed for the study did not provide any training at all. Practitioners seeking to develop virtual team training may consider including content on 'sensitivity to cultural differences' and 'creating a team mission statement' as a comparison of effective compared to less effective training demonstrated that more effective virtual team training incorporated these aspects (Rosen et al., 2006).

At present there is limited available research on organizational interventions designed to address workplace cyber harassment. Nonetheless researchers have suggested that CREW (civility, respect and engagement at work) training could be adapted by practitioners to cover cyber communication (Giumetti et al., 2013; Park et al., 2015). CREW is an intervention designed to reduce acceptance of uncivil behaviors whereby workgroups attend weekly meetings to learn civil ways of interacting. It may be particularly relevant for volatile virtual teams, as it aims to help work units identify ways to reduce incivility (Osatuke, Moore, Ward, Dyrenforth and Belton, 2009). This is done by helping workgroups develop and implement their own intervention, rather than imposing an intervention upon them. Organizations struggling with cyber incivility may consider the method as studies demonstrate that CREW training reduces face-to-face incivility, absenteeism and burnout (Leiter, Laschinger, Day and Oore, 2011; Osatuke et al., 2009).

A further strategy concerns the implementation of organizational policies that prohibit negative ICT communication. West et al. (2014) interviewed human resources professionals on the effectiveness of cyberbullying policies. All interviewees reported that their organization had some form of workplace harassment policy, however respondents noted that even when their organizations policy covered cyberbullying, training was needed to heighten awareness. Organizations may also consider developing social media policies. The popular press has coined the term 'Facebook Fired' to refer to instances where employees have been sacked from their jobs as a result of posting on social media (Schmidt and O'Connor, 2015). To prevent such occurrences, Schmidt and O'Connor (2015) suggest organizations craft clear policies that do not overly restrict employees and include examples of prohibited wrongdoing.

\section{SUMMARY OF FINDINGS}

The key findings from this review are summarized in Figure 1 for each of the four dimensions. In addition to this figure, we now provide a summary of the 
key findings across all dimensions.

\section{Organizational outcomes}

As noted in this review, some demands created by ICTs are just plain detrimental to organizational performance. Cyber forms of harassment can deplete employee resources, reduce positive affect and increase distress (Giumetti, et al; Park et al, 2015) Poor communication in general can create misunderstandings which may cause frustration, stress, (Day et al, 2010) poor decisions (Jackson and Van den Hooff, 2012) or weaker interpersonal relationships (Byron 2008). In addition, employees can take from 64 seconds to 15 minutes to recover from an email interruption and reengage in their primary task (Jackson et al., 2001; Van Solingen et al., 1998), and knowledge workers might lose up to 28 minutes of their workday because of interruptions (Gupta and Sharda, 2008). Similarly, those being overloaded by emails experience reduced task coordination and productivity (Dabbish and Kraut, 2006; Karr-Wisniewski and Lu, 2010).

However, some demands act as a "double-edged sword" (Dén-Nagy, 2014) by increasing organizational performance under certain circumstances, but at a personal cost. On one hand, performance might be increased by interruptions carrying work-related information or a higher volume of work-related emails (Mano and Mesch, 2010). Individuals having access to ICT outside working hours might feel empowered by devices such as BlackBerrys resulting in a greater sense of satisfaction, professionalism, productivity, autonomy, control (Cavazotte et al., 2014; Day et al., 2012; Matusik and Mickel, 2011). On the other hand, ICT response expectations have been found to increase physical and cognitive burnout, health-related absenteeism and cause poorer sleep quality (Barber and Santuzzi, 2015). The $24 / 7$ availability increases work-life conflict and work-life imbalance (Derks et al., 2015; Diaz et al., 2012; Matusik and Mickel, 2011; Wright et al., 2014), outcomes which have been associated with job burnout (Wright et al., 2014). This paradox is intriguing, as employees are often aware of these tradeoffs and often embrace the introduction of some these technologies rather than resisting it (Cavazotte et al., 2014; Mazmanian, 2013).

\section{Possible interventions}

To tackle some of these outcomes, this review discussed several possible interventions suggested in previous studies. First, training interventions could be implemented to improve email quality, clarity and efficiency (Jackson et al., 2006), to tackle email overload (Soucek and Moser, 2010), to reduce cyber incivility (Giumetti et al., 2013) or to prepare for ICT-related work-life conflict (Leonardi et al., 2010). The review discussed some content proposed in these training interventions such as role-playing activities or commented examples.

Organizational policies could also be designed to mitigate the negative outcomes identified. The impact of these demands on productivity should be assessed in the organization (Al-Dabbagh et al., 2014), then collectively discussed (Barber and Santuzzi, 2015). Researchers have suggested that organizations should then decide on which behaviors to promote and which to restrict (Matusik and Mickel, 2011). Depending on the results of internal surveys, discussions and debates, policies could be designed; indeed the review discussed some examples such as guidelines or restrictions. 
Figure 1: Summary of key findings

\begin{tabular}{|l|}
\hline RESPONSE EXPECTATIONS \\
Organizational outcomes \\
- Some interruptions might increase \\
performance (Mano and Mesch, \\
2010; Addas and Pinsonneault, 2015; \\
Speier et al., 2003). \\
- Yet it takes time to recover from \\
interruptions (Jackson et al., 2001; \\
Gupta and Sharda, 2008). \\
And these response expectations \\
can lead to detrimental health \\
effects (Barber and Santuzzi, 2015). \\
Possible interventions \\
- Increase the message checking \\
frequency (Gupta et al., 2011, \\
Jackson et al., 2001). \\
Avoid synchronous messages if not \\
needed (Gupta et al., 2013). \\
Assess interruptions impact on \\
productivity within the organization \\
(Al-Dabbagh et al., 2014).
\end{tabular}

\section{CONSTANT AVAILABILITY Organizational outcomes}

- Constant availability can also cause work-life imbalance and conflicts (Derks et al., 2015; Diaz et al., 2012, Wright et al., 2014).

- Although employees are aware of these problems, they often choose to remain constantly available and feel more productive and professional by doing so (Cavazotte et al., 2014; Matusik and Mickel, 2011).

\section{Possible interventions}

- Help employees to deal with worklife conflicts with support groups, collective discussions or training (Leonardi et al., 2010; Barber and Santuzzi, 2015).

- Discourage or ban work communication beyond working hours (Wright et al., 2014).

\begin{tabular}{|l|}
\hline WORKLOAD \\
Organizational outcomes \\
- The more emails, the longer the \\
workday and the greater the feelings \\
of overload (Barley et al., 2011). \\
Receiving plenty of work-related \\
emails can increase work \\
effectiveness but also stress and \\
distress (Mano and Mesch, 2010). \\
Information overload can lead to \\
lowered productivity (Karr- \\
Wisniewski and Lu, 2010; Dabbish \\
and Kraut, 2006). \\
Possible interventions \\
Train employees to improve their \\
use of emails in order to deal with \\
email overload (Burgess et al., 2005; \\
Jackson and Lichtenstein, 2011). \\
Circulate guidelines encouraging \\
users to limit the amount of emails \\
they send (Soucek and Moser, 2010). \\
\hline
\end{tabular}

\begin{tabular}{|l|}
\hline \multicolumn{1}{|c|}{ POOR COMMUNICATION } \\
Organizational outcomes \\
- Misunderstood messages can lead to \\
frustration, stress and poor \\
decisions (Jackson and Van den \\
Hoof, 2012). \\
Cyber incivility increases distress, \\
decreases task performance and \\
reduces energy (Giumetti et al., \\
2013; Park et al., 2015). \\
Cyberbullying is linked to increased \\
turnover intentions, anxiety and \\
frustration (Baruch, 2005; Hong et \\
al., 2014). \\
Possible interventions \\
- Train employees to write clearer \\
emails (Jackson et al., 2006). \\
Train employees about civility, \\
respect and engagement at work \\
(Osatuke et al., 2009). \\
Implement cyberbullying policies \\
(West et al., 2014).
\end{tabular}


Finally, we discussed what can be done at an individual level. For example, managers could act as role models in terms of constant availability by refraining from sending messages beyond working hours (Derks et al., 2015) or individuals could change the setup of their email software in order to tackle ICT interruptions (Addas and Pinsonneault, 2015; Jackson et al., 2006).

Having summarized the key findings across the four dimensions, we now discuss their implications for research as well as key gaps in this literature.

\section{IMPLICATIONS FOR RESEARCH}

The current digital environments that individuals face at work places demands on them that are potentially detrimental to performance and well-being (Tarafdar et al., 2015b). Although research is beginning to examine some of these issues, there is a need to theoretically structure these 'dark side' ICT related phenomena in order to (1) understand what we already know about them and to (2) provide directions for future research. This paper tackles these two objectives from the point of view of ICT communication in organizations. Specifically it accomplishes the following objectives. One, current studies examine different kinds of impacts of ICT such as loss of employee control (Mazmanian et al., 2013) and poor performance (Tarafdar et al., 2015a). However these studies look at disparate characteristics of and expectations from IT use without providing an integrated understanding of these demands. By focusing on four key characteristics of ICT communication, this paper looks at the different demands and their possible organizational consequences in an integrated manner, thus providing theoretical structure to this phenomenon. Two, we identify the organizational interventions that can offset the negative consequences of ICT. In doing so, we theoretically link the demands from ICT, their organizational consequences and possible organizational interventions. This takes the literature forward by explicitly focusing on interventions rather than just on the demands of ICT or their consequences. Finally, in reporting systematically on the state of the current literature we are able to suggest directions where future research should direct its efforts to further develop the area of negative consequences of ICT communication.

Specifically, the review highlighted the double-edged nature of ICT in that some productivity benefits also come at a personal cost. This paradox could be further investigated if studies on ICT and productivity were to systematically include well-being-related outcomes. Looking at both productivity and wellbeing emphasizes that organizational effectiveness is about both people and performance. This paper also discussed innovative and clear suggestions for practice identified by other researchers. Future studies could follow the example of some of the papers cited to improve their recommendations for practice. We suggest that the 'implications for practice' section should be considered key here. These suggestions for practice could then be investigated in other research studies such as quasi experiments. For example, studies have suggested that training interventions might be designed to tackle email overload, and Burgess and colleagues (2005) successfully built upon this suggestion by designing an actual training intervention and by evaluating its outcomes. Some interventions discussed in this paper such as email guidelines or interventions on cyber 
harassment are yet to be investigated in practice. These studies have much to bring to both research and practice. Finally, most ICT methods leave behind a trail of their existence, which presents opportunities for researchers as it may be possible to identify objective instances of overload, interruption or cyber harassment and determine their subsequent impact on organizational effectiveness. In this paper, we reviewed experimental research which demonstrated that interacting with an uncivil supervisor was associated with lower task performance (Giumetti et al., 2013). This suggests that there is potential for field studies that can use objective indicators of overload or interruption (e.g. number or volume of emails received) and how they are linked with subsequent indicators of employee health and performance.

\section{IMPLICATIONS FOR PRACTICE AND CONCLUSIONS}

We have reviewed four aspects of ICT communication that affect the health and performance of employees: expectations that they should respond immediately to communications, 24-7 availability, increased workload and the potential for poor communication. There are a number of potential interventions that could minimize the nefarious aspects of these downsides. Regarding response expectations, employees could be trained to let recipients know whether their email is a priority so that recipients can determine when they need to respond and can then prioritize their workload. Furthermore, longer message checking intervals can enable people to get on with their primary work, rather than constantly tuning in. Also employees should be discouraged from 'reply to all' and not cc people who really have no 'need to know'. In terms of 'constant availability', both employee and employer have a responsibility to set personal and organizational guidelines about accessing email and other forms of ICT outside of working hours. The research evidence is clear that this 24-7 culture is damaging people's health and relationships outside, as well as their productivity. The reactions from those concerned about this have led some employers to shut down servers at the weekends and to create 'email free' times during the week. The very least employers should do is create guidelines and diktats which encourage people to be 'email free' during non-work time unless absolutely necessary. In respect of the impact of ICT on workloads, we would encourage people to severely limit the 'ccing' of people into emails, let the recipient know the priority of the email, train people on email filtering or archiving, develop a detailed email policy and encourage employees to check their emails less frequently and not in the evening or on holiday unless absolutely necessary. Finally, there are a range of practical implications to minimize the risk of misunderstandings. Many organizations are now stopping email communications within the same building to encourage colleagues to meet face to face, as this can minimize communication difficulties and promote team building. Guidelines should also emphasize that difficult conversations (e.g., about job loss, promotions, performance management) should not be done by any form of ICT. Another worrying trend alluded to earlier is the increase in cyberbullying, so training that addresses this (i.e. the CREW programme), should be considered by employers if they identify this as an increasing problem area. As much as possible when it comes to difficult or complex or nuanced 
conversations they should be done by face to face communications or at the very least video conferencing.

In summary, senior management should be responsible for ICT guidelines, employer expectations, encouraging better work-life balance and minimizing the potential negative effects of poor use of ICT. As Einstein once wrote about technology "I fear the day that technology will surpass our human interaction the world will have a generation of idiots". Technology can be an enormously important tool but we need to manage it rather than it manage us!

\section{REFERENCES}

Addas, S., Pinsonneault, A., 2015. The many faces of information technology interruptions: a taxonomy and preliminary investigation of their performance effects. Info Systems J 25, 231-273. doi:10.1111/isj.12064

Al-Dabbagh, B., Sylvester, A., Scornavacca, E., 2014. To connect or disconnect-that is the question: ICT self-discipline in the 21st century workplace. ACIS.

Ayyagari, R., Grover, V., Purvis, R., 2011. Technostress: Technological Antecedents and Implications. MIS Quarterly 35, 831-858.

Bailey, D.E., Kurland, N.B., 2002. A review of telework research: findings, new directions, and lessons for the study of modern work. Journal of Organizational Behavior 23, 383-400. doi:10.1002/job.144

Barber, L.K., Santuzzi, A.M., 2015. Please Respond ASAP: Workplace Telepressure and Employee Recovery. Journal of Occupational Health Psychology 20, 172-189. doi:10.1037/a0038278

Barley, S.R., Meyerson, D.E., Grodal, S., 2011. E-mail as a Source and Symbol of Stress. Organization Science 22, 887906.
Baruch, Y., 2005. Bullying on the net: Adverse behavior on e-mail and its impact. Information \& Management, 42, 361-371. doi:10.1016/j.im.2004.02.001

BBC News., 2014. Managers 'work extra day per week in unpaid overtime'. Retrieved from http://www.bbc.co.uk/news/business28220312.

Bellotti, V., Ducheneaut, N., Howard, M., Smith, I., Grinter, R.E., 2005. Quality Versus Quantity: E-Mail-Centric Task Management and Its Relation With Overload. Human-Computer Interaction 20, 89-138. doi:10.1207/s15327051hci2001\&2_4

Boswell, W.R., Olson-Buchanan, J.B., 2007. The use of communication technologies after hours: The role of work attitudes and work-life conflict. Journal of Management 33, 592-610.

Burgess, A., Jackson, T., Edwards, J., 2005. Email training significantly reduces email defects. International Journal of Information Management 25, 71-83. doi:10.1016/j.ijinfomgt.2004.10.004

Byron, K., 2008. Carrying too heavy a load? The communication and miscommunication of emotion by email. Academy of Management Review, 33, 309-327. doi:10.5465/AMR.2008.31193163

Cavazotte, F., Heloisa Lemos, A., Villadsen, K., 2014. Corporate smart phones: professionals' conscious engagement in escalating work connectivity. New Technology, Work and Employment 29, 72-87.

Cramton, C. D., Orvis, K. L., Wilson, J. M., 2007. Situation invisibility and attribution in distributed collaborations. Journal of Management, 33 , 525-546. doi: $10.1177 / 0149206307302549$

Currie, J., Eveline, J., 2011. E-technology and work/life balance for academics with young children. Higher Education, 62, 533-550. doi: 10.1007/s10734-010-9404-9

Daft, R. L., Lengel, R. H., 1986. Organizational information requirements, media richness and structural design. 
Management Science, 32, 554-571.

Dabbish, L.A., Kraut, R.E., 2006. Email overload at work: an analysis of factors associated with email strain, in: Proceedings of the 2006 20th Anniversary Conference on Computer Supported Cooperative Work. ACM, pp. 431-440.

Day, A., Scott, N., Kelloway, E. K., 2010. Information and communication technology: Implications for job stress and employee well-being. In $\mathrm{P}$. Perrewe., D. Ganster (Eds), New developments in theoretical and conceptual approaches to job stress: Research in occupational stress and well being 8, 317-350. Burlington, VT: Emerald Group Publishing Ltd.

Day, A., Paquet, S., Scott, N., Hambley, L., 2012. Perceived information and communication technology (ICT) demands on employee outcomes: The moderating effect of organizational ICT support. Journal of Occupational Health Psychology 17, 473.

Dén-Nagy, I., 2014. A double-edged sword?: a critical evaluation of the mobile phone in creating work-life balance. New Technology, Work and Employment 29, 193-211.

Derks, D., van Duin, D., Tims, M., Bakker, A.B., 2015. Smartphone use and work-home interference: The moderating role of social norms and employee work engagement. Journal of Occupational and Organizational Psychology 88, 155177. doi:10.1111/joop.12083

Diaz, I., Chiaburu, D.S., Zimmerman, R.D., Boswell, W.R., 2012. Communication technology: Pros and cons of constant connection to work. Journal of Vocational Behavior 80, 500-508. doi:10.1016/j.jvb.2011.08.007

Eden, D., 2001. Vacations and other respites: Studying stress on and off the job. In C. L. Cooper., I. T. Robertson (Eds.), International review of industrial and organizational psychology, 121-146. Chichester, England: Wiley.

Farley, S., Coyne, I., Sprigg, C., Axtell, C., Subramanian, G., 2015. Exploring the impact of workplace cyberbullying on trainee doctors. Medical education, 49, 436-443. DOI: 10.1111/medu. 12666

Ford, D. P., 2013. Virtual harassment: media characteristics' role in psychological health. Journal of Managerial Psychology, 28, 408-428. http://dx.doi.org/10.1108/JMP-12-20120398

Friedman, R. A., \& Currall, S. C., 2003. Conflict Escalation: Dispute Exacerbating Elements of Email Communication. Human Relations, 56, 1325-1347. doi: $10.1177 / 00187267035611003$

Gajendran, R.S., Harrison, D.A., 2007. The Good, the Bad, and the Unknown About Telecommuting: Meta-Analysis of Psychological Mediators and Individual Consequences. Journal of Applied Psychology 92, 1524-1541.

Giumetti, G.W., Hatfield, A.L., Scisco, J.L., Schroeder, A.N., Muth, E.R., Kowalski, R.M., 2013. What a rude e-mail! Examining the differential effects of incivility versus support on mood, energy, engagement, and performance in an online context. Journal of Occupational Health Psychology 18, 297-309. doi:10.1037/a0032851

Gupta, A., Li, H., Sharda, R., 2013. Should I send this message? Understanding the impact of interruptions, social hierarchy and perceived task complexity on user performance and perceived workload. Decision Support Systems 55, 135-145. doi:10.1016/j.dss.2012.12.035

Gupta, A., Sharda, R., 2008. SIMONE: A Simulator for Interruptions and Message Overload in Network Environments. International Journal of Simulation and Process Modelling 4, 237-247.

Gupta, A., Sharda, R., Greve, R.A., 2011. You've got email! Does it really matter to process emails now or later? Information Systems Frontiers 13, 637653.

doi:http://dx.doi.org/10.1007/s10796010-9242-4

Heatherington, W., Coyne, I., 2014. Understanding individual experiences of cyberbullying encountered through 
work. International Journal of Organization Theory and Behaviour, 17, 163-192.

Hong, J. C., Chien-Hou, L., Hwang, M. Y., Hu, R. P., Chen, Y. L., 2014. Positive affect predicting worker psychological response to cyber-bullying in the hightech industry in Northern Taiwan. Computers in Human Behavior, 30, 307-314. doi:10.1016/j.chb.2013.09.011

Jackson, T.W, Dawson, R., Wilson, D., 2001. The cost of email interruption. Journal of Systems and Information Technology 5, 81-92.

Jackson, T.W., Burgess, A., Edwards, J., 2006. A simple approach to improving email communication. Communications of the ACM 49, 107-109.

Jackson, T.W., Dawson, R.J., Wilson, D., 2003. Breaking Bad Habits: The Negative Effect of Email and Instant Messaging on the Workplace", in: Khosrow-Pour, M. (Ed.), Information Technology \& Organisations: Trends, Issues, Challenges \& Solutions. Idea Group, Hershey, USA, pp. 399-401.

Jackson, T.W., Lichtenstein, S., 2011. Optimising e-mail communication: the impact of seminar- and computerbased training. International Journal of Internet and Enterprise Management 7, 197-216. doi:10.1504/IJIEM.2011.039915

Jackson, T. W., Van den Hooff, B., 2012. Understanding the Factors that Effect Information Overload and Miscommunication within the Workplace. Journal of Emerging Trends in Computing and Information Sciences, 3, 1240-1252.

Karr-Wisniewski, P., Lu, Y., 2010. When more is too much: Operationalizing technology overload and exploring its impact on knowledge worker productivity. Computers in Human Behavior 26, 1061-1072.

Kock, N., 2004. New Theory of ComputerMediated Communication Based on Darwinian Evolution, Organization Science, 15, 327-348.

Kokkalis, N., Köhn, T., Pfeiffer, C., Chornyi, D., Bernstein, M.S., Klemmer, S.R., 2013.
EmailValet: managing email overload through private, accountable crowdsourcing, in: Proceedings of the 2013 Conference on Computer Supported Cooperative Work. ACM, pp. 1291-1300.

Kushlev, K., Dunn, E.W., 2015. Checking email less frequently reduces stress. Computers in Human Behavior 43, 220 228. doi:10.1016/j.chb.2014.11.005

Leiter, M. P., Laschinger, H., Day, A., Oore, D., 2011. The impact of civility interventions on employee social behavior, distress, and attitudes. Journal of Applied Psychology, 96, 1258-1274.

Leonardi, P.M., Treem, J.W., Jackson, M.H., 2010. The Connectivity Paradox: Using Technology to Both Decrease and Increase Perceptions of Distance in Distributed Work Arrangements. Journal of Applied Communication Research 38, 85-105. doi:10.1080/00909880903483599

Li, H., Gupta, A., Luo, X., Warkentin, M., 2011. Exploring the impact of instant messaging on subjective task complexity and user satisfaction. Eur J Inf Syst 20, 139-155. doi:10.1057/ejis.2010.59

Lim, V. K., Teo, T. S., 2009. Mind your Emanners: Impact of cyber incivility on employees' work attitude and behavior. Information \& Management, 46, 419425.

Lundberg, U., Lindfors, P., 2002. Psychophysiological reactions to telework in female and male whitecollar workers. Journal of Occupational Health Psychology, 7, 354-364.

Madden, M. and Jones, S. (2008), "Networked workers", Pew Research Center, Washington, D.C., 24 September, available at: www.pewinternet.org/2008/09/24/netwo rked-workers/

Mano, R.S., Mesch, G.S., 2010. E-mail characteristics, work performance and distress. Computers in Human Behavior 26, 61-69. doi:10.1016/j.chb.2009.08.005

Mark, G., Voida, S., Cardello, A., 2012. A pace 
not dictated by electrons: an empirical study of work without email, in: Proceedings of the SIGCHI Conference on Human Factors in Computing Systems. ACM, pp. 555-564.

Matusik, S.F., Mickel, A.E., 2011. Embracing or embattled by converged mobile devices? Users' experiences with a contemporary connectivity technology. Human Relations 64, 1001-1030. doi:10.1177/0018726711405552

Mazmanian, M., 2013. Avoiding the Trap of Constant Connectivity: When Congruent Frames Allow for Heterogeneous Practices. Academy of Management Journal 56, 1225-1250. doi:10.5465/amj.2010.0787

Mazmanian, M., Orlikowski, W.J., Yates, J., 2013. The Autonomy Paradox: The Implications of Mobile Email Devices for Knowledge Professionals. Organization Science 24, 1337-1357. doi:10.1287/orsc.1120.0806

Office for National Statistics., 2009. Statistical Bulletin: E-commerce and ICT 2009. Retrieved from: www.statistics.gov.uk/pdfdir/ecom1110. pdf

Osatuke, K., Moore, S. C., Ward, C., Dyrenforth, S. R., Belton, L., 2009. Civility, Respect, Engagement in the Workplace (CREW): Nationwide organization development intervention at Veterans Health Administration. Journal of Applied Behavioral Science, 45 , 384-410. doi: $10.1177 / 0021886309335067$

Panteli, N., Dawson, P., 2001. Video conferencing meetings: changing patterns of business communication. New Technology, Work and Employment, 16, 88-99. DOI: 10.1111/1468-005X.00079

Park, Y., Fritz, C., Jex, S. M., 2011. Relationships between work-home segmentation and psychological detachment from work: the role of communication technology use at home. Journal of Occupational Health Psychology, 16, 457-467.

Park, Y., Fritz, C., Jex, S. M., 2015. Daily Cyber Incivility and Distress The Moderating
Roles of Resources at Work and Home. Journal of Management. Available online ahead of print. doi:10.1177/0149206315576796

Palvalin, M., Lönnqvist, A., \& Vuolle, M. (2013). Analysing the impacts of ICT on knowledge work productivity. Journal of Knowledge Management, 17, 545557. doi: http://dx.doi.org/10.1108/JKM03-2013-0113

Porath, C. L., Pearson, C. M., 2010. The cost of bad behavior. Organizational Dynamics, 39, 64-71. doi:10.1016/j.orgdyn.2009.10.006

Privitera, C., Campbell, M. A. 2009. Cyberbullying: The new face of workplace bullying?. CyberPsychology \& Behavior, 12, 395-400. doi:10.1089/cpb.2009.0025

Rosen, B., Furst, S., Blackburn, R., 2006. Training for virtual teams: An investigation of current practices and future needs. Human Resource Management, 45, 229-247. DOI: $10.1002 / \mathrm{hrm} .20106$

Schmidt, G. B., O'Connor, K. W., 2015. Fired for Facebook: Using NLRB guidance to craft appropriate social media policies. Business Horizons. Available online ahead of print.

Snyman, R., Loh, J. M., 2015. Cyberbullying at work: The mediating role of optimism between cyberbullying and job outcomes. Computers in Human Behavior, 53, 161-168. doi:10.1016/j.chb.2015.06.050

Soucek, R., Moser, K., 2010. Coping with information overload in email communication: Evaluation of a training intervention. Computers in Human Behavior, Online Interactivity: Role of Technology in Behavior Change 26, 1458-1466. doi:10.1016/j.chb.2010.04.024

Speier, C., Vessey, I., Valacich, J.S., 2003. The effects of interruptions, task complexity, and information presentation on computer-supported decision-making performance. Decision Sciences 34, 771-797.

Sproull, L., Kiesler, S., 1986. Reducing social context cues: Electronic mail in 
Organizational Communication, Management Science, 32, 1492-1512. doi: $10.2307 / 2631506$

Sumecki, D., Chipulu, M., Ojiako, U., 2011. Email overload: Exploring the moderating role of the perception of email as a "business critical" tool. International Journal of Information Management 31, 407-414. doi:10.1016/j.ijinfomgt.2010.12.008

Tarafdar, M., Gupta, A., Turel, O., 2015a. The Dark Side of Information Technology Use: Introduction to the Special Issue and a Research Framework. Info Systems J 25, 161-170. doi:10.1111/isj.12070

Tarafdar, M., Pullins, E.B., Ragu-Nathan, T.S., 2015b. Technostress: negative effect on performance and possible mitigations. Info Systems J 25, 103-132. doi:10.1111/isj.12042

Tarafdar, M., Qiang Tu, Ragu-Nathan, B.S., Ragu-Nathan, T.S., 2007. The Impact of Technostress on Role Stress and Productivity. Journal of Management Information Systems 24, 301-328.

The Metro., 2014. Mozilla chief Brendan Eich resigns: Can you criticise your boss on Twitter? Retrieved from: http://metro.co.uk/2014/04/03/mozillachief-brendan-eich-resigns-can-youcriticise-your-boss-on-twitter-4688332/

The Weekend Australian., 2007. More weekends spent in office. Careers, p. 11.

Van Solingen, R., Berghout, E., Van Latum, F., 1998. Interrupts: just a minute never is. IEEE software 97-103.

Waller, A.D., Ragsdell, G., 2012. The impact of e-mail on work-life balance. Aslib Proceedings 64, 154-177. doi:http://dx.doi.org/10.1108/000125312 11215178

Walther, J. B., Boos, M., Jonas, K. J. 2002. Misattribution and attributional redirection in distributed virtual groups. HICSS. Proceedings of the 35th Annual Hawaii International Conference on System Sciences. IEEE. doi: 10.1109/HICSS.2002.994427

Weatherbee, T. G., Kelloway, E. K., 2006. A Case of Cyberdeviancy: CyberAggression in the Workplace. In E. K. Kelloway., J. Barling., J. J. Hurrell (Eds.), Handbook of Workplace Violence. 445-487. Thousand Oaks, CA: Sage Publications, Inc.

West, B., Foster, M., Levin, A., Edmison, J., Robibero, D., 2014. Cyberbullying at Work: In Search of Effective Guidance. Laws, 3, 598-617.

Wright, K.B., Abendschein, B., Wombacher, K., O’Connor, M., Hoffman, M., Dempsey, M., Krull, C., Dewes, A., Shelton, A., 2014. Work-Related Communication Technology Use Outside of Regular Work Hours and Work Life Conflict The Influence of Communication Technologies on Perceived Work Life Conflict, Burnout, Job Satisfaction, and Turnover Intentions. Management Communication Quarterly 28, 507-530. doi:10.1177/0893318914533332 